\title{
Personalized therapy tests for the monitoring of chronic lymphocytic leukemia development
}

\author{
MAŁGORZATA ROGALIŃSKA ${ }^{1}$, PAWEŁ GÓRALSKI ${ }^{2}$, JERZY Z. BŁOŃSKI ${ }^{3}$, \\ PAWEŁ ROBAK ${ }^{4}$, JAN BARCISZEWSKI ${ }^{5}$, ANETA KOCEVA-CHYŁA ${ }^{6}$, \\ HENRYK PIEKARSKI ${ }^{2}$, TADEUSZ ROBAK ${ }^{3}$ and ZOFIA M. KILIANSKA ${ }^{1}$

\begin{abstract}
Departments of ${ }^{1}$ Cytobiochemistry and ${ }^{2}$ Physical Chemistry, University of Lodz, Lodz 90-236; Departments of ${ }^{3}$ Hematology and ${ }^{4}$ Experimental Hematology, Medical University of Lodz, Lodz 90-419; ${ }^{5}$ Institute of Bioorganic Chemistry, Polish Academy of Science, Poznan 61-704;

${ }^{6}$ Department of Medical Biophysics, University of Lodz, Lodz 90-236, Poland
\end{abstract}

Received June 4, 2016; Accepted November 17, 2016

DOI: $10.3892 / \mathrm{ol} .2017 .5725$

\begin{abstract}
There is individual variation in the course of disease development and response to therapy of patients with chronic lymphocytic leukemia (CLL). Novel treatment options for CLL include a new generation of purine analogs, antibodies and inhibitors of specific cell signaling pathways, which typically induce apoptosis or necrosis. A prospective analysis of patient blood samples revealed that a combination of four tests allowed the most appropriate and effective type of treatment to be selected prior to drug administration, and for the analysis of leukemic cell sensitivity to anticancer drug(s) during disease development. The comparative analysis of blood from the stable and progressive form of CLL in an individual patient revealed diversity in the response to anticancer agents. CLL peripheral blood mononuclear cells were incubated with cladribine + mafosfamide $(\mathrm{CM})$, fludarabine + mafosfamide, $\mathrm{CM}+$ rituximab, rituximab alone ( $\mathrm{Rit}$ ) or kinetin riboside (RK). A combination of cell viability, differential scanning calorimetry (DSC) profiles of nuclear preparations and poly(ADP-ribose) polymerase 1 (PARP-1) protein expression analysis of the leukemic cells was performed to evaluate the anticancer effects of the tested agents during CLL development. The results of the present study indicate that such studies are effective in determining the most appropriate anticancer drug and could monitor disease progression on an individual level. In addition, the results of the current study suggest that CLL progression leads to diversification of the cellular drug response. The most efficient apoptosis inducer for the patient was purine analog RK when the disease was stable, while the
\end{abstract}

Correspondence to: Dr Małgorzata Rogalińska, Department of Cytobiochemistry, University of Lodz, 141-143 Pomorska Street, Lodz 90-236, Poland

E-mail: mrogalin@biol.uni.lodz.pl

Key words: chronic lymphocytic leukemia, personalized therapy, anticancer drugs, cell signal inhibitors, apoptosis
$\mathrm{CM}$ combination was the most effective agent for the progressive form of disease.

\section{Introduction}

Although progress has been made in the diagnostics and treatment for chronic lymphocytic leukemia (CLL), this type of neoplasm remains incurable, similarly to other types of cancer at an advanced stage. The primary problem faced by hematologists in the treatment of CLL is the coexistence of quiescent and cycling cell populations, which exhibit different responses to anticancer drugs (1-5). Additional issues include disease heterogeneity and differences in the time of disease development. These factors complicate therapeutic decisions $(4,5)$.

Genetic aberrations and epigenetic modifications in neoplastic lymphocytes are associated with variations in the course of the disease, which may affect the response to therapy (6-9). Numerous studies have identified factors that may affect the anticancer response and disease prognosis, such as genetic aberrations, alterations at a micro (mi)RNA level and epigenetic modifications (6-14). Currently available diagnostic markers for CLL, including specific genetic aberrations, are insufficient to provide a clear indication of the correct anticancer treatment choice $(9,15)$. Individual differences in the expression of the factors that regulate apoptosis, cell signaling and stimulate the cellular microenvironment may result in nonstandard and unfavorable responses to anticancer therapy.

Previous studies have investigated potential therapeutic agents for the ability to induce apoptosis (14) and inhibit proteins involved in cell signaling $(16,17)$. Due to the complexity of metabolic signaling pathways (18), choosing an effective therapeutic regimen for CLL patients remains difficult. The heterogeneity of CLL and the growing number of novel therapeutic options, such as immunochemotherapy, immunomodulators and cell signaling inhibitors, emphasizes the requirement to improve the methods for determining individual sensitivity to different therapies, in order to allow the selection of the optimal treatment $(15,19,20)$. Therapeutic decisions are additionally complicated by the variation in disease development $(4,5,21)$. Therefore, additional studies are 
required with respect to patients with CLL that are 'refractory', or resistant, to drugs, and those who are in the active stage of the disease (9).

A previous study of 28 patients with CLL (15), and a previously published case report (20), revealed a correlation between apoptosis induction potential of cells isolated from peripheral blood of untreated CLL patients to the subsequent application of anticancer drugs (cladribine, mafosfamide, fludarabine, rituximab or their combinations). The present study compared two blood analyses from a young previously-untreated adult female. The first blood sample was taken during the stable form of the disease, and the second was obtained 18 months subsequently during the active form of disease. Peripheral blood leukemic mononuclear cells (PBMCs) were isolated, and used to evaluate the cytotoxicity and proapoptotic activity of the tested drugs (described below). In addition, the subsequent clinical response to the applied treatment was recorded. A comparative cytometric analysis of cell viability, apoptosis rate, differential scanning calorimetry (DSC) profiles of nuclear preparations and poly(ADP-ribose) polymerase 1 (PARP-1) protein expression of the leukemic cell samples was performed as described previously $(15,20)$. The ability of CLL cells to initiate apoptosis following exposure to cladribine + mafosfamide $(\mathrm{CM})$, fludarabine + mafosfamide (FM), CM + rituximab ( $\mathrm{RCM})$, rituximab alone (Rit) or kinetin riboside $(\mathrm{RK})$ was investigated.

\section{Materials and methods}

Patients and treatment. The present study was approved by the Ethics Committee of the Medical University of Lodz (Lodz, Poland; approval no. RNN/143/10/KE). Informed consent was provided for the use of patient data in this study. The first sample was collected in July 2012 during stable disease when the patient was 22 years old and the second sample was taken 18 months later in January 2014, during progressive disease. The white blood cell count was 120,000 cells/ $\mu 1$ in 2012 and 657,000 cells $/ \mu 1$ in 2014. CLL diagnosis of this patient was performed in Hematology Department of Medical University of Lodz at the Copernicus Memorial Hospital (Lodz, Poland) according to standard clinical, immunological and cytological criteria from the International Workshop on Chronic Lymphocytic Leukemia $(1,22,23)$.

Following blood sample analysis, fludarabine + cyclophosphamide administration was commenced, according to standard pharmacological conditions (22). The patient received fludarabine (Teva Pharmaceutical Industries, Ltd., Petah Tikva, Israel) at a dose of $25 \mathrm{mg} / \mathrm{m}^{2}$ combined with cyclophosphamide (Baxter International, Inc., Deerfield, IL, USA) at $250 \mathrm{mg} / \mathrm{m}^{2}$ for 3 days intravenously (FC regimen), every 28 days for 6 cycles. Clinical response to the treatment following 6 cycles of drug administration was evaluated as previously described $(22,24)$. Following this treatment, the patient achieved complete remission.

Isolation of CLL cells and incubation with drugs. PBMCs were isolated from the two blood samples using Histopaque-1077 (Sigma-Aldrich; Merck Millipore, Darmstadt, Germany) according to the manufacturer's instructions. The obtained cells were washed with PBS and cultured at a final density of $3 \times 10^{6}$ cells/ml in RPMI-1640 medium (Cytogen $\mathrm{GmbH}$, Zgierz, Poland) containing $10 \%$ fetal calf serum (Biowest, Nuaillé, France), 2 mM L-glutamine (Sigma-Aldrich; Merck Millipore, Darmstadt, Germany), $100 \mathrm{U} / \mathrm{ml}$ penicillin and $100 \mu \mathrm{g} / \mathrm{ml}$ streptomycin (Biowest). The leukemic PBMCs were subsequently incubated with anticancer agents. Cells were incubated with CM, FM, RCM, Rit or RK as described previously $(25,26)$. Cladribine was supplemented in dose of $50 \mu \mathrm{g} / \mathrm{ml}$, mafosfamide at $1 \mu \mathrm{g} / \mathrm{ml}$, Rit at $20 \mu \mathrm{g} / \mathrm{ml}$, fludarabine at $20 \mu \mathrm{M}$ and kinetin riboside at $20 \mu \mathrm{M}$. A group not treated with any agents was used as a control. The leukemic cells were incubated without any agents (control) or treated with these anticancer agents for $48 \mathrm{~h}$ at $37^{\circ} \mathrm{C}$ with $5 \% \mathrm{CO}_{2}$.

Cladribine (Biodrybin), fludarabine and rituximab were obtained from the Institute of Biotechnology and Antibiotics (Warsaw, Poland), fludarabine was obtained from Teva Pharmaceutical Industries, Ltd. and Rit was obtained from Roche (Basel, Switzerland). Mafosfamide was obtained from Baxter Oncology GmbH (Frankfurt, Germany) or purchased from Niomech IIT GmbH (Bielefeld, Germany). The kinetin riboside was obtained from the Institute of Bioorganic Chemistry (Polish Academy of Science, Poznan, Poland).

Cell viability and apoptosis assay. The leukemic cells were incubated in culture medium, with or without (the control) drug treatment. The fractions of viable and apoptotic cells following $48 \mathrm{~h}$ of exposure were determined by Vybrant Apoptosis assay kit \#4 (Molecular Probes; Thermo Fisher Scientific, Inc., Waltham, MA, USA), as described previously (15).

Preparation of the nuclear fraction and cell lysates. Following a $48 \mathrm{~h}$ incubation period, the control and drug-treated CLL cells were rinsed with PBS and homogenized in isotonic sucrose solution [5 mM MgCl $2,0.5 \%$ Triton X-100, $50 \mathrm{mM}$ Tris- $\mathrm{HCl}$ (pH 7.4), protease inhibitors] as described previously (26). The homogenate was then centrifuged at $1,000 \times \mathrm{g}$ for $10 \mathrm{~min}$ to obtain the nuclear fraction. The leukemic cells were subsequently lysed in a buffer containing $10 \mathrm{mM}$ Tris- $\mathrm{HCl}(\mathrm{pH} 7.5)$, $300 \mathrm{mM} \mathrm{NaCl}, 1 \%$ Triton X-100, 2 mM MgCl $2,0.1 \mathrm{M}$ dithiothreitol and a mixture of protease inhibitors, as described previously (26). The protein content was measured by standard Lowry method.

Immunoblotting. The protein samples (40 $\mu \mathrm{g} /$ lane) were separated by SDS-PAGE on an $8 \%$ gel and blotted onto an Immobilon-P polyvinylidene fluoride membrane. Protein loading and transfer were confirmed by Ponceau S staining. The membranes were blocked with $5 \%$ non-fat powdered milk dissolved in TBS [10 mM Tris- $\mathrm{HCl}(\mathrm{pH} 7.5)$ and $150 \mathrm{mM} \mathrm{NaCl}$ ] for $1 \mathrm{~h}$ at room temperature. Subsequent to washing in TBS containing $0.05 \%$ Tween-20, the blots were incubated with a primary antibody directed against PARP-1 (cat. no. sc-7150; dilution, 1:2,000; Santa Cruz Biotechnology, Inc., Dallas, TX, USA), myeloid leukemia cell differentiation protein (Mcl-1; dilution, 1:3,000) or B-cell lymphoma 2 (Bcl-2; dilution, 1:2,000) produced by Santa Cruz Biotechnology, Inc. An alkaline phosphatase-conjugated secondary antibody was then used, as previously described (26). 
A

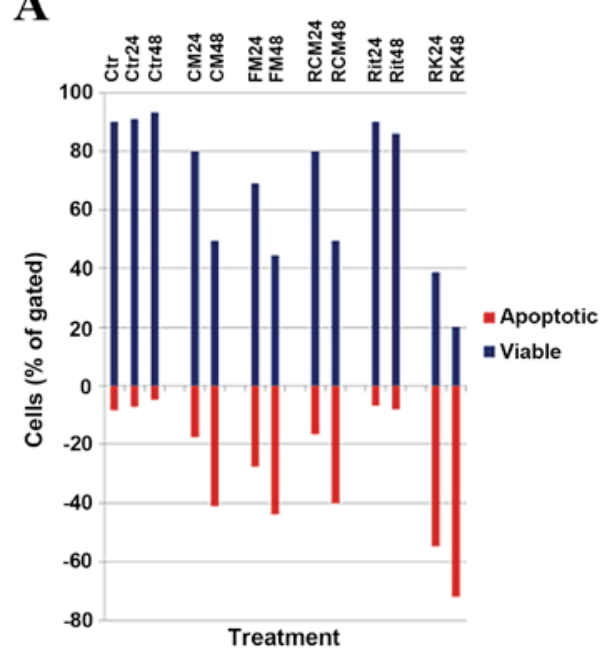

C

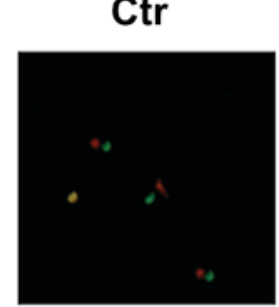

RCM

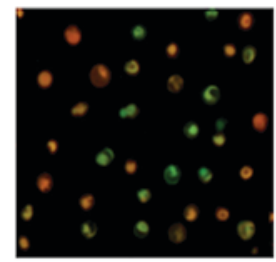

CM

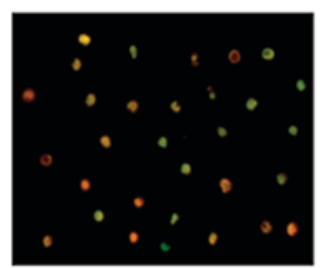

Rit

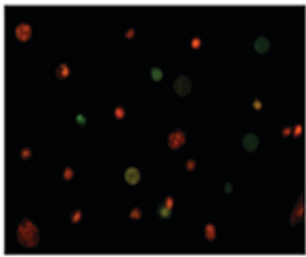

B

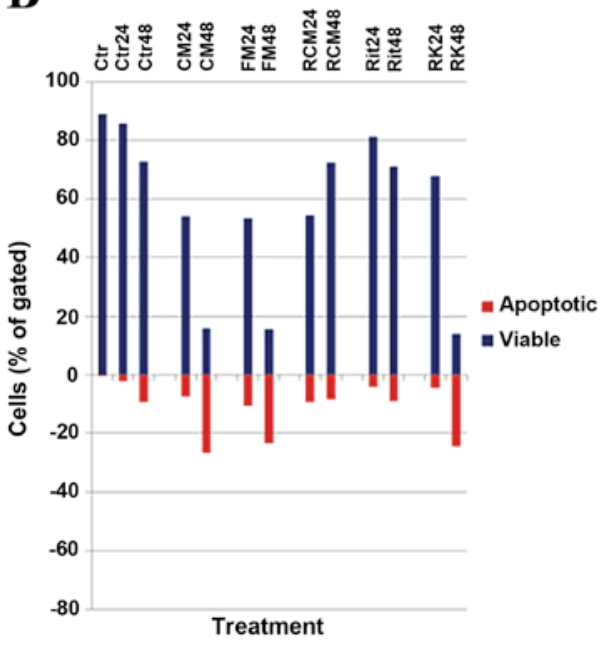

FM

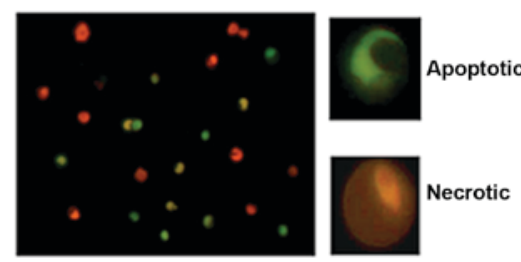

RK

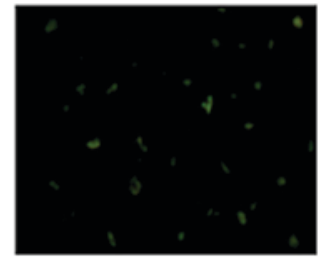

Figure 1. Cell viability, apoptosis and necrosis $48 \mathrm{~h}$ following exposure to anticancer agents. Comparative analysis of the viability and apoptosis rate of CLL cells isolated during the (A) stable and (B) progressive form of the disease. (C) Representative images of apoptotic and necrotic CLL cells (magnification, x400). Numerous cells with the typical morphological features of apoptosis (green) and necrosis (red), cells were observed. CLL; chronic lymphocytic leukemia; Ctr, control; CM, cladribine + mafosfamide; FM, fludarabine + mafosfamide; RCM, CM + rituximab; Rit, rituximab alone; RK, kinetin riboside.

$D S C$. Nuclear fraction preparations of the control and treated cells were prepared for calorimetric tests according to the protocol used by Almagor and Cole (27). The nuclear fraction samples were transferred into pans and hermetically sealed. Calorimetric experiments were performed using a TG-DSC 111 calorimeter (Setaram, Caluire, France). A temperature of $20-120^{\circ} \mathrm{C}$ and a scanning rate of $5^{\circ} \mathrm{C} / \mathrm{min}\left(0.083^{\circ} \mathrm{C} / \mathrm{s}\right)$ was used, as previously reported $(28,29)$.

Monitoring of apoptosis and necrosis. The amount of apoptotic and necrotic cells up to $48 \mathrm{~h}$ following drug exposure were monitored using a fluorescence microscope (IX70; Olympus Corporation, Tokyo, Japan; magnification, x400). The cells were suspended in PBS at a density of $\sim 1 \times 10^{6}$ cells $/ \mathrm{ml}$, incubated for $\sim 20 \mathrm{~min}$ (room temperature, in the dark) with YO-PRO-1 and propidium iodide (PI), and transferred onto microscopic slides. The YO-PRO-1 fluorescent dye selectively labels apoptotic cells green. PI stains late apoptotic and necrotic cells red. The stained cells were classified as apoptotic and necrotic, as previously described (30).

\section{Results}

CLL cells indicate susceptibility to anticancer drugs. The results of the cell viability assay, DSC, immunoblotting and proteolytic degradation of the apoptotic marker PARP-1 highlighted the differences between the classic stable and progressive forms of the disease. The most marked changes were observed in the fractions of living and apoptotic cells (Fig. 1), as well as in the nuclear fraction profiles analyzed by DSC (Fig. 2) and PARP-1 expression/proteolytic degradation (Fig. 3). Additionally, a reduced number of apoptotic leukemic cells was observed during progressive disease (Fig. 1B). In addition, variation was observed in the proteolytic degradation of PARP-1 in leukemic cells incubated with RK, in the stable (Fig. 3A) and progressive forms of CLL (Fig. 3B). These results demonstrate the differences in CLL cell sensitivity to anticancer agents between the stable and progressive forms of the disease.

The combination of tests useful in personalized therapy for CLL (15) may be valuable in monitoring aspects of CLL 

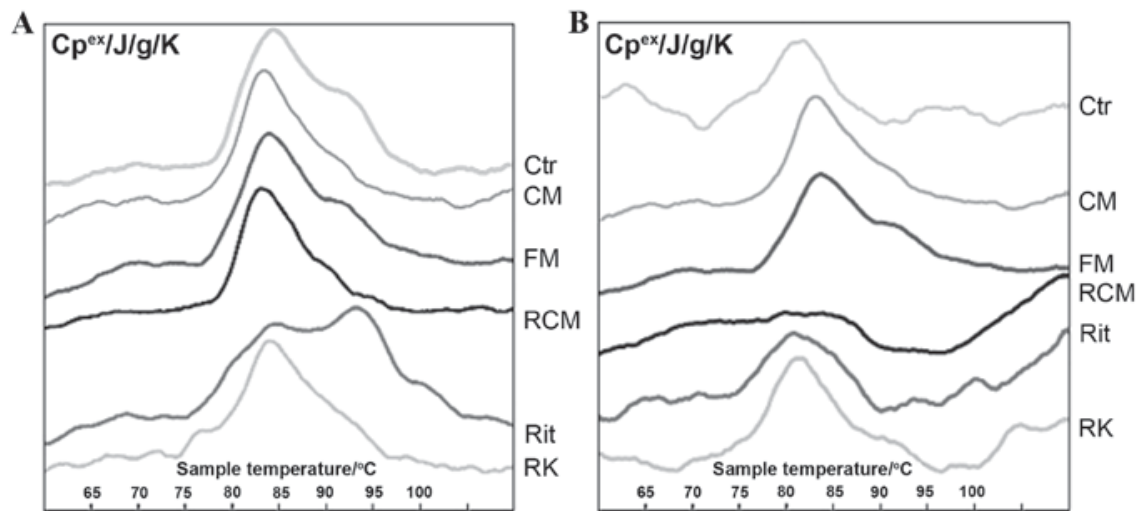

Figure 2. Differential scanning calorimetry profiles of the nuclear fraction of chronic lymphocytic leukemia cells $48 \mathrm{~h}$ following exposure to anticancer agents. (A) Stable form of the disease. (B) Progressive form of the disease. Ctr, control; CM, cladribine + mafosfamide; FM, fludarabine + mafosfamide; RCM, CM + rituximab; Rit, rituximab alone; RK, kinetin riboside.

$\mathbf{A}$

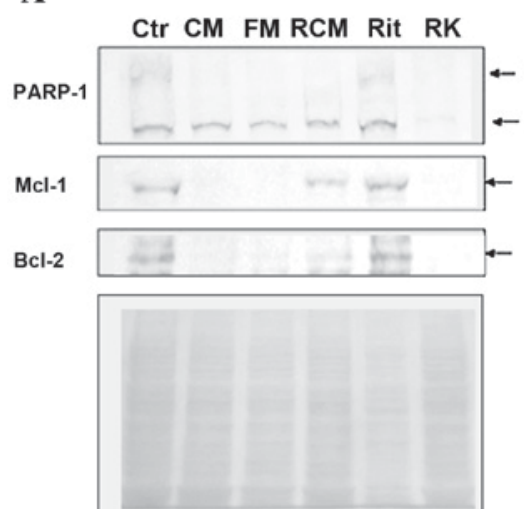

B

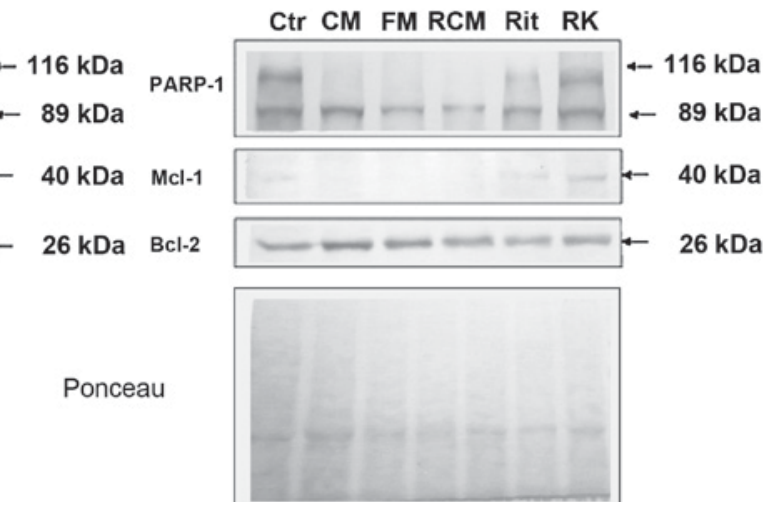

Figure 3. Changes in PARP-1, Mcl-1 and Bcl-2 protein expression in chronic lymphocytic leukemia cell lysates $48 \mathrm{~h}$ following exposure to anticancer agents. (A) Stable form of the disease. (B) Progressive form of the disease. PARP-1, Poly (ADP-ribose) polymerase 1; Mcl-1, myeloid leukemia cell differentiation protein; Bcl-2, B-cell lymphoma 2; Ctr, control; CM, cladribine + mafosfamide; FM, fludarabine + mafosfamide; RCM, CM + rituximab; Rit, rituximab alone; RK, kinetin riboside.

progression, particularly the diversity in potential of leukemic cells to induce apoptosis during disease development. In addition, these tests could track the changes in chromatin structure that occur when cell proliferation is induced, which is indicative of the progressive form of the disease (Fig. 2B). These results contrast with the cell features identified during the characteristic stable form of the disease (Figs. 1A-3A).

Comparative tests aid disease monitoring. A comparative analysis was performed between the blood samples obtained during stable and progressive CLL. The comparison of the results from the four tests performed revealed that the stable form of the disease exhibited the typical features of apoptosis induction, whereas the progressive disease revealed a high proliferative index and elevated leukocytosis score.

In the stable form of the disease, all anticancer agents tested induced a higher level of PBMC apoptosis compared with the control group (Fig. 1A). However, when the same drugs were applied to the leukemic PBMCs from progressive CLL, a reduced level of apoptosis was observed (Fig. 1B). Changes in corresponding cell viability were noted subsequent to incubation with CM, FM and RCM. The PBMCs exhibited a lack of activity when cultured with Rit. Notably, RK demonstrated the greatest apoptotic induction in stable (Figs. 1A and 3A) and progressive leukemic cells (Figs. 1B and 3B). The DSC profiles of the nuclear fraction confirmed the presence of diverse changes in CLL chromatin conformation, resulting from exposure to the tested agents (Fig. 2). Considerable differences in leukemic cell reactivity were observed in cell samples incubated with Rit, with the cells appearing resistant to Rit in the stable form of the disease. Previous studies have reported that in progressive CLL, additional thermal transition occurs at $\sim 95 \pm 5^{\circ} \mathrm{C}(15,19,20,26,28,29)$, which decreases in the case of the apoptotic activity. The presence of a thermal transition at $95^{\circ} \mathrm{C}$ following CLL incubation with $\geq 1$ of the agents indicates that the leukemic cells in the stable form of the disease are resistant to Rit.

Proteolytic degradation of the apoptotic marker PARP-1 was observed in cells undergoing drug-induced apoptosis (Fig. 3). However, although PARP-1 was cleaved when resting leukemic cells were incubated with RK, this was not the case in the progressive form of the disease. In addition, PARP-1 was not cleaved in cells following exposure to Rit, suggesting that Rit is not effective, particularly in the stable form of the disease. The proapoptotic degradation of the antiapoptotic proteins Mcl-1 and Bcl-2 corresponded with other results of the present study, demonstrating induction of apoptosis. Notably, in the progressive form of CLL, a decreased apoptosis level was found to be associated with $\mathrm{Bcl}-2$ expression. 
PBMCs from the progressive form of CLL were analyzed for apoptosis and necrosis cells (Fig. 1C), in order to investigate which anticancer drug(s) were inducers of apoptosis in CLL cells. In the studied samples, from the CLL patient, CM and FM demonstrated strong activity as apoptotic inducers in leukemic PBMCs, indicating that they may be effective in the treatment of progressive CLL. In contrast, the most effective drug for the treatment of the stable form of disease in the patient studied was RK (Figs. 1A-3A), a novel purine analog with high apoptosis potential.

\section{Discussion}

The diagnostics and treatment of patients with CLL is typically based upon the standard clinical, immunological and cytological criteria of the International Workshop on Chronic Lymphocytic Leukemia $(1,22,23)$. Hematological diagnostic factors include specific genetic alterations and protein expression, such as that of $\beta 2$-microglobulin, $\zeta$-chain-associated protein kinase 70 and cluster of differentiation $38(8,9,21)$. At present, there is insufficient knowledge to predict the most effective treatment for CLL on an individual level. Cell comprise of a complicated network of factors involved in gene expression, cellular metabolism, and signaling pathways associated with trafficking and apoptosis $(14,31)$. Despite growing research, it remains impossible to screen for the potential response to therapy at an individual genetic level, due to the high variation in genetic and metabolic profiles (15). The presence of epigenetic changes, such as the degree of protein methylation or acetylation, can complicate the interpretation of genetic information. A recent study published by Houldsword et al (32) investigated the association between genomic alterations and risk stratification in patients with CLL. In addition, analyses of the expression or mutation status of specific genes may be insufficient for the prediction of the outcome of in vivo therapy (15).

The number of studies investigating the metabolic signaling occurring within CLL cells is increasing (18), but the issue is complicated by the involvement of the tumor microenvironment and the regulation of gene expression by epigenetic changes. Therefore, a promising approach for selecting the optimal treatment for an individual patient is to incubate the leukemic cells of the patient with a set of potential anticancer drugs in vitro. This test would allow the detection of the response of the leukemic cells to anticancer drugs to be predicted prior to in vivo administration. Previous studies have demonstrated that these tests may be of value in predicting the sensitivity of cancer cells to drugs $(15,20)$. The results of a previous study (15), indicated that this test aided in the selection of treatment and subsequent response of patients with a p17 deletion, a marker of poor patient prognosis.

The findings of the present study indicate that the incubation of leukemic PBMCs with a set of anticancer agents can be used to monitor the development of CLL. In addition, this method may reveal the potential response to certain drugs prior to their administration in vivo, thus enabling personalized treatment of patients (33). The combination of tests performed in the present study appears to be sufficient for identifying leukemic cell sensitivity to anticancer agents. However, the identification of novel CLL treatments remains important to increase treatment options and improve patient outcomes (34).
The results of present study indicate that PBMCs from the stable and active forms of CLL exhibit different responses to anticancer agents. Notably, the most efficient apoptosis inducer was found to be the purine analog RK for the stable form of the disease, while CM was the most effective type in the progressive form.

\section{Acknowledgements}

The authors of the present study would like to thank Dr Aneta Rogalska for her help in the capturing of cell images. The present study was supported by the Polish National Science Centre (grant no. 2011/01/B/NZ4/01702).

\section{References}

1. Oscier D, Dearden C, Erem E, Fegan C, Follows G, Hillmen P, Illidge T, Matutes E, Milligan DW, Pettitt A, et al: Guidelines on the diagnosis, investigation and management of chronic lymphocytic leukaemia. Br J Haematol 159: 541-564, 2012.

2. Hallek M: Chronic lymphocytic leukemia: 2015 Update on diagnosis, risk and stratification and treatment. Am J Hematol 90: 446-460, 2015

3. Cramer P, Hallek M and Eichhorst B: State-of-the-art treatment and novel agents in chronic lymphocytic Leukemia. Oncol Res Treat 39: 25-32, 2016.

4. Rodriquez-Vicente AE, Diaz MG and Hernándes-Rivas JM: Chronic lymphocytic leukemia: A clinical and molecular heterogenous disease. Clin Genet 206: 49-62, 2013.

5. Klein U and Dalla-Favera R: Germinal centres: Role in B-cell physiology and malignancy. Nat Rev Immunol 8: 22-33, 2008.

6. Zenz T and Huber W: Mutational landscape and complexity in CLL. Blood 126: 2078-2079, 2015.

7. Oakes CC, Seifert M, Assenov Y, Gu L, Przekopowitz M, Ruppert AS, Wang Q, Imbusch CD, Serva A, Koser SD, et al: DNA methylation dynamics during $B$ cell maturation underlie a continuum of disease phenotypes in chronic lymphocytic leukemia. Nat Genet 48: 253-264, 2016.

8. Puiggros A, Blanco G and Espinet B: Genetic abnormalities in chronic lymphocytic leukemia: Where we are and where we go. Biomed Res Int 2014: 435983. 2014.

9. Bulian P, Rossi D, Forconi F, Del Poeta G, Bertoni F, Zucca E, Montillo M, Pozzato G, D'Arena G, Efremov DG, et al: IGHV gene mutational status and $17 \mathrm{p}$ deletion are independent molecular predictors in a comprehensive clinical-biological prognostic model for overall survival prediction in chronic lymphocytic leukemia. J Transl Med 10: 18, 2012.

10. Rogalinska $M$ and Kilianska ZM: Targeting Bcl-2 in CLL. Curr Med Chem 19: 5109-5115, 2012.

11. Freeman CL, Morschhauser F, Sehn L, Dixon M, Houghton R, Lamy T, Fingerle-Rowson G, Wassner-Fritsch E, Gribben JG, Hallek M, et al: Cytokine release in patients with CLL treated with obinutuzumab and possible relationship with infusion-related reactions. Blood 126: 2646-2649, 2015.

12. Martín-Subero JI, López-Otin C and Campo E: Genetic and epigenetic basis of chronic lymphocytic leukemia, Curr Opin Hematol 20: 362-368, 2013.

13. Burger JA and Gribben JG: The microenvironment in chronic lymphocytic leukemia (CLL) and other B cell malignancies: Insight into disease biology and new targeted therapies. Semin Cancer Biol 24: 71-81, 2014.

14. Chen R and Plunkett W: Strategy to induce apoptosis and circumvent resistance in chronic lymphocytic leukemia. Best Pract Res Clin Haematol 23: 155-166, 2010.

15. Rogalińska M, Błoński JZ, Góralski P, Wawrzyniak E, Hartman M, Rogalska A, Robak P, Koceva-Chyła A, Piekarski H, Robak T and Kiliańska ZM: Relationship between in vitro drug sensitivity and clinical response of patients to treatment in chronic lymphocytic leukemia. Int J Oncol 46: 1259-1267, 2015.

16. Burger JA, Tedeschi A, Barr PM, Robak T, Owen C, Ghia P, Bairey O, Hillmen P, Bartlett NL, Li J, et al: Ibrutinib as initial therapy for patients with chronic lymphocytic leukemia. N Engl J Med 373: 2425-2437, 2015. 
17. Falchi L, Baron JM, Orlikowski CA and Ferrajoli A: BCR signaling inhibitors: An overview of toxicities associated with ibrutinib and idelalisib in patients with chronic lymphocytic leukemia. Mediterr J Hematol Infect Dis 8: e2016011, 2016.

18. Rogalinska M: The role of mitochondria in cancer induction, progression and changes in metabolism. Mini Rev Med Chem 16: 524-530, 2016

19. Rogalinska M, Blonski J, Goralski P, Komina O, Kazmierczuk A, Wawrzyniak E, Kotkowska A, Robak P, Piekarski H, Robak T, et al: Usefulness of differential scanning calorimetry for monitoring ex vivo the changes in responses of CLL cells to anticancer drugs: Development of personalized therapy. Blood (ASH Annual Meeting Abstracts) 116: Abstract 4635, 2010.

20. Rogalińska M, Franiak-Pietryga I, Błoński JZ, Góralski P, Maciejewski H, Janus A, Robak P, Mirowski M, Piekarski H, Robak T and Kiliańska ZM: Toward personalized therapy for chronic lymphocytic leukemia: DSC and cDNA microarray assessment of two cases. Cancer Biol Ther 14: 6-12, 2013.

21. Zenz T, Gribben JG, Hallek M, Döhner H, Keating MJ and Stilgenbauer S: Risk categories and refractory CLL in the era of chemoimmunotherapy. Blood 119: 4101-4107, 2012.

22. Cheson BD, Bennet JM, Grever M, Kay N, Keating MJ, O'Brien S and Rai KR: National cancer institute sponsored working group guidelines for chronic lymphocytic leukemia: Revised guidelines for diagnosis and treatment. Blood 87: 4990-4997, 1996.

23. Wierda WG: Updates to the management of chronic lymphocytic leukemia. J Natl Compr Canc Natw 13 (Suppl 5): S662-S665, 2015.

24. Robak T, Blonski JZ, Gora-Tybor J, Jamroziak K, Dwilewicz-Trojaczek J, Tomaszewska A, Konopka L, Ceglarek B, Dmoszynska A, Kowal M, et al: Cladribine alone and in combination with cyclophosphamide or cyclophosphamide plus mitoxantrone in the treatment of progressive chronic lymphocytic leukemia: Report of a prospective, multicenter, randomized trial of Polish Adult Leukemia Group (PALG CLL2). Blood 108: 473-479, 2006.

25. Kobylinska A, Bednarek J, Blonski JZ, Hanausek M, Walaszek Z Robak T and Kilianska ZM: In vitro sensitivity of B-cell chronic lymphocytic leukemia to cladribine and its combinations with mafosfamide and/or mitoxantrone. Oncol Rep 16: 1389-1395, 2006.
26. Rogalińska M,Blonski JZ,Komina O,Góralski P,Zołnierczyk JD, Piekarski H, Robak T, Kiliańska ZM and Wesierska-Gadek J: R-roscovitine (Seliciclib) affects CLL cells more strongly than combinations of fludarabine or cladribine with cyclophosphamide: Inhibition of CDK7 sensitizes leukemic cells to caspase-dependent apoptosis. J Cell Biochem 109: 217-235, 2010.

27. Almagor M and Cole RD: Differential scanning calorimetry of nuclei as a test for the effects of anticancer drugs on human chromatin. Cancer Res 49: 5561-5566, 1989.

28. RogalińskaM,Góralski P,Kobylińska A,Bloński JZ,Hanausek M, Walaszek Z, Piekarski H, Robak T and Kiliańska ZM: Changes in leukemic cell nuclei revealed by differential scanning calorimetry. Leuk Lymphoma 46: 121-128, 2005.

29. Goralski P, Rogalinska M, Blonski JZ, Pytel E, Robak T, Kiliańska ZM and Piekarski H: The differences in thermal profiles between normal and leukemic cells exposed to anticancer drug evaluated by differential scanning calorimetry. J Therm Anal Calorim 118: 1339-1344, 2014

30. Gasiorowski B, Brokos A, Kulma A, Ogorzałek A and Skórkowska K: A comparison of the methods applied to detect apoptosis in genotoxically damaged lymphocytes cultured in the presence of four antimutagens. Cell Biol Mol Lett 6: 141-159, 2001.

31. Burger JA and Chiorazzi N: B cell receptor signaling in chronic lymphocytic leukemia. Trends Immunol 34: 592-601, 2013.

32. Houldsworth J, Guttapalli A, Thodima V, Yan XJ, Mendiratta G, Zielonka T, Nanjangud G, Chen W, Patil S, Mato A, et al: Genomic imbalance defines three prognostic groups for risk stratification of patients with chronic lymphocytic leukemia. Leuk Lymphoma 55: 920-928, 2014.

33. Rogalińska M and Kiliańska ZM: Personalized therapy versus targeted therapy, differences in the meaning. Global J Res Anal 4: 5-8, 2015.

34. Podhorecka M, Macheta A, Chocholska S, Bojarska-Junak A, Szymczyk A, Goracy A, Dmoszynska A and Hus M: Danazol induces apoptosis and cytotoxicity of leukemic cells alone and in combination with purine nucleoside analogs in chronic lymphocytic leukemia. Ann Hematol 95: 425-435, 2016. 